\title{
ON THE MECHANISM OF REACTIVE ADSORPTION OF DIBENZOTHIOPHENE ON ORGANIC WASTE DERIVED CARBONS
}

\author{
CO Ania ${ }^{1,2}$, JB Parra ${ }^{1, *}$, A Arenillas ${ }^{1}$, F Rubiera ${ }^{1}$, TJ Bandosz ${ }^{2}$, JJ Pis ${ }^{1}$ \\ ${ }^{1}$ Instituto Nacional del Carbón, CSIC, Apartado 73, 33080 Oviedo, Spain \\ ${ }^{2}$ Chemistry Department, The City College of New York, New York, NY 10031, USA. \\ * Corresponding author E-mail: jbparra@incar.csic.es
}

\begin{abstract}
The mechanism of reactive adsorption of dibenzothiophene (DBT) on a series of modified carbons derived from recycled PET was investigated. The influence of the oxygen functionalities of the adsorbent on the DBT adsorption capacity was explored. The results revealed that adsorption of DBT on activated carbons is governed by two types of contributions: physisorption on the microporous network of the carbons and chemisorption. Introduction of surface acidic groups enhanced the performance of the carbons as a result of their specific interactions with DBT. The nature of the acidic groups is a decisive factor in the selectivity of the reactive adsorption process.
\end{abstract}

Keywords: Desulfurization, dibenzothiophene, reactive adsorption, PET waste.

\section{INTRODUCTION}

Organosulfur compounds in gasoline and diesel not only have a detrimental effect on the environment but their also interfere in modern technological processes [1]. Thus increasing concerns on the air quality and new environmental regulations urged the petroleum refining industry to produce cleaner products by reducing the sulfur level from their major products, diesel and gasoline [2-5]. Desulfurization of liquid fuels in the refineries is usually achieved by catalytic hydrodesulfurization (HDS) at elevated temperatures and pressures $[6,7]$. To comply with the new regulations, this approach faces an important challenge: the removal of polyaromatic sulfur-containing compounds without an unavoidable and significant capital investment. One way to avoid the increased costs is to use other technologies, such as adsorption, which operates at ambient temperature and pressure making this approach an attractive field of research [8-12]. The challenge here is the development of 
adsorbents with a high adsorption capacity and selectivity for sulfur containing species over other aromatic and olefinic compounds.

In recent research, we have reported that dibenzothiophene (DBT) adsorption on carbon materials could be improved via incorporating adequate active sites on the carbon surface $[13,14]$. Strong oxidation of commercial carbons lead to an enhancement in DBT removal, whereas unexpectedly high adsorptive capacities were obtained on a polystyrene-based salts-derived carbons loaded with several transition metals with a catalytic activity. However the mechanism of adsorption or the nature of the reactive adsorption remained not clear at that stage.

Therefore, in this work we investigate the mechanism of desulfurization via reactive adsorption on activated carbons obtained from PET waste. Understanding the mechanism of adsorption is critical to improve the performance of carbons for deep desulfurization of liquids fuels. The aim is to enhance the adsorption forces and selectivity and/or to induce reactive adsorption via incorporation of acidic oxygen-containing functionalities on the carbon matrix. The effects of changes in the surface chemistry of the activated carbons were studied in relation to the changes in the capacity of the adsorbents. The approach used includes the synthesis of the carbon materials, the characterization of the surface functionalities and the study of their DBT adsorption capacity. The modifications in the textural properties of the modified carbons with respect to the parent carbon were also studied.

\section{EXPERIMENTAL}

\section{Materials}

The procedure for the preparation of the PET-derived carbons has been previously described [15]. In order to increase the surface heterogeneity, PL carbon was oxidized with $\left(\mathrm{NH}_{4}\right)_{2} \mathrm{~S}_{2} \mathrm{O}_{8}$ (PLS) and $20 \%$ v/v nitric acid (PLN) following the procedure described elsewhere [16]. The oxidized samples were washed with distilled water in a Soxhlet apparatus and dried at $120^{\circ} \mathrm{C}$.

\section{Adsorption of DBT from solution}

Adsorption of DBT was carried out at room temperature in a stirred batch system as described elsewhere [14]. In brief, different amounts of carbons (from $25 \mathrm{mg}$ to $1 \mathrm{~g}$ ) were added to bottles containing $40 \mathrm{~cm}^{3}$ of the sulfur-containing solution with an initial concentration of $1000 \mathrm{ppmw}$ of DBT (ca. 178 ppmw of S) dissolved in hexane. The covered bottles were allowed to shake for $72 \mathrm{~h}$ at 
a constant temperature. After equilibration the concentration in the solution was determined using a UV spectrophotometer at the corresponding wavelength. The equilibrium data was fitted to the socalled Langmuir-Freundlich (LF) single solute isotherm [17]. The fitting range was from 0 to $250 \mathrm{mg}$ of S per gram of activated carbon (recalculated from its content in DBT).

\section{Textural characterization}

Textural characterization was carried out by measuring the $\mathrm{N}_{2}$ adsorption isotherms at $-196^{\circ} \mathrm{C}$. Before the experiments, the samples were outgassed under vacuum at $120^{\circ} \mathrm{C}$ overnight. The isotherms were used to calculate the specific surface area, $\mathrm{S}_{\mathrm{BET}}$, total pore volume, $\mathrm{V}_{\mathrm{T}}$, and pore size distributions. The pore size distributions were evaluated using density functional theory (DFT) [18].

\section{Thermal analysis}

Temperature programmed desorption (TPD) experiments were carried out in a thermogravimetric system under an argon flow rate of $50 \mathrm{~cm}^{3} \mathrm{~min}^{-1}$, at a heating rate of $15^{\circ} \mathrm{C} \min { }^{-1}$, up to a final temperature of $1000^{\circ} \mathrm{C}$. A quadrupole mass spectrometer (MS), and an infrared spectrometer (FTIR), linked to the thermobalance were used for evolved gas analysis. To avoid secondary reactions, a probe was placed very close to the sample crucible of the thermobalance in the direction of the gas flow. The gas lines between the TG and the MS/FTIR were heated to $180^{\circ} \mathrm{C}$ in order to avoid cold points. For each experiment, about $35 \mathrm{mg}$ of a ground carbon sample was used.

\section{XRF analysis}

X-Ray Fluorescence analysis was applied to study the content of sulfur in the carbons, before and after DBT exposure. The amount of sulfur was determined based on calibration curves prepared from a carbonaceous matrix. For this purpose, SPECTRO Model 300T Benchtop Multi-Channel Analyzer from ASOMA Instruments, Inc. was used at the following conditions: voltage $9.0 \mathrm{kV}$, current $280 \mu \mathrm{A}$, count time $100 \mathrm{sec}$, warm-up $3 \mathrm{~min}$, reference temperature was $20^{\circ} \mathrm{C}$ and background conditions: lower ROI 3200, upper ROI $5750 \mathrm{keV}$.

\section{RESULTS AND DISCUSSION}

Figure 1 shows the DBT adsorption isotherms on the PET-derived carbons which belong to the L type in the Giles classification [19]. Based on their shape it is seen that i) there is preferential adsorption of DBT over the solvent (hexane) on the active sites (no strong solvent competition), and ii) the amount 
adsorbed steadily increases at low doses of adsorbent, although the saturation limit is not attained. Table 1 provides a compilation of the adsorptive capacities obtained from fitting the experimental data to the LF equation, along with the correlation coefficients. The adsorptive capacity of a microporous commercial carbon (BP from Calgon) is also shown for comparison. The results show that the PET-derived carbons have favorable features for the removal of DBT. The adsorption capacities are comparable to and/or larger than those reported in the literature for zeolites or carbons aerogels $[11,12,20]$. Such large capacities are linked to the adequate textural properties of these materials since, as indicated elsewhere [13, 14,] narrow microporosity of activated carbons acts as active centers for DBT adsorption. The amount of sulfur in the carbons after DBT exposure was measured by XRF and recalculated in terms of weight of DBT. The results show a good agreement for PL, PLN and BP with the expected amount of sulfur adsorbed, calculated from the adsorption capacities obtained from fitting the experimental data to the LF model.

After modification of the as-received sample, PL, the adsorptive capacities increased and the best performance obtained for the sample treated with ammonium persulfate PLS. The beneficial effect of oxygen functionalities on DBT removal has also been reported by Jiang and coworkers after oxidation of activated carbons with sulfuric acid [20]. The effect of oxidation on the increase in the specific DBT adsorption is also corroborated by the decrease in the exponent $n$ of the LF equation (from an initial value of 0.38 for PL to 0.30 for PLS), indicating that formation of surface functionalities creates new active sites with different energies toward DBT adsorption than those in the case of the original carbon [21].

Previous studies on commercial activated carbons with different textural properties showed that adsorption capacity is rather related to the pore size distribution than to the extent of the surface area [13]. Despite the fact that a linear correlation between the capacity and the volume of narrow micropores $(\mathrm{w}<0.7 \mathrm{~nm}$ ) was reported, the surface heterogeneity of the carbon matrix plays also an important role and thus both texture and chemical composition of carbons could not be considered independently. For this reason, we have investigated activated carbons with similar textural properties but different surface functionalities, in order to throw some light on the mechanism of adsorption. By changing the surface chemistry, without much altering the textural properties, we might be able to 
deal exclusively and independently with the benefits of oxygen-containing groups for the abatement of DBT, and to acquire a proper understanding of the mechanism of reactive adsorption induced by the functionalities of the carbon. To attain this goal the surface chemistry of the raw carbon (PL) was carefully modified by wet oxidation in mild conditions with two oxidizing agents: ammonium persulfate and nitric acid. It can be clearly seen in Table 2 that oxidation did not appreciably change the textural properties of the initial carbon. The measurement of the $\mathrm{pH}$ of the carbons in a water suspension revealed that the majority of the groups created are of an acidic nature.

The nature of these groups was evaluated by TPD/MS and TPD/FTIR (Figure 2). The profiles showed desorption of $\mathrm{CO}$ above $500{ }^{\circ} \mathrm{C}$ and $\mathrm{CO}_{2}$ starting from $200{ }^{\circ} \mathrm{C}$. According to the literature [22] the $\mathrm{CO}_{2}$ evolved at low temperatures can be assigned to carboxylic groups, whereas the $\mathrm{CO}$ evolving at higher temperatures comes from weakly acidic and neutral groups [22].

The relative intensities of both $\mathrm{CO}$ and $\mathrm{CO}_{2}$ peaks are larger for PLN than for PLS sample. Nevertheless, the profiles are qualitatively identical, which indicates that the oxygenated groups created are the same in both samples, although in smaller amount in PLS, in accordance to its higher oxygen content (Table 2). It is interesting that the TPD/FTIR profiles showed evolution of $\mathrm{SO}_{2}$ in PLS sample (Figure 2) at two temperatures. Although the nature of sulfur surface complexes has not been extensively reported, this suggests that two types of sulfur functionalities are present. These might be assigned to thiols (low temperature peak) and more stable sulfoxides, sulfones and/or sulfides (C-S-C) [23]. Sulfur was not located on the external surface since it was not detected by XPS.

Based on the above, the surface chemistry was successfully modified by incorporating diverse groups of acidic nature via choosing different reagents and adjusting the operating conditions. Moreover, the same type of oxygen-containing functionalities was created in both modified samples, and modification with ammonium persulfate led to the incorporation of sulfur species, likely acidic based on the surface $\mathrm{pH}$, to the carbon matrix (Table 2).

Since the carbons studied have similar textural characteristics, it is clear that the increase in the DBT retention observed for the oxidized carbons has to be exclusively related to the presence of surface functionalities. Linking this finding with the nature of these groups, the enhancement in adsorption 
may be attributed to several factors, including either favorable interactions between the acidic sites of the carbon and the basic structure of DBT molecule, and/or on sulfur-oxygen interactions.

When one tries to correlate the adsorption capacities with the oxygen content of the carbons, the relationship found is not straightforward (Figure 3). This suggests that rather than the total amount of oxygen, the strength of acidic sites should be considered. In fact, a much better dependence exists between the uptake and the $\mathrm{pH}$ or the density of acidic sites -evaluated as the milliequivalents of $\mathrm{HCl}$ consumed per gram of adsorbent-. Thus, the acidic groups coming from the sulfur incorporated to PLS carbon are also accounted for the correlation. A similar trend had also been observed previously in commercial activated carbon [13]. This confirms the hypothesis of acidic groups (either oxygen or sulfur-based) as active sites for DBT adsorption. This also explains the fact that despite the largest number of oxygen functionalities of PLN sample, evidenced by the largest relative intensities in the TPD data (Figure 2), the DBT uptake is smaller for that sample than that for PLS.

The DTG profiles for the samples after DBT adsorption showed two peaks (Figure 4). As described elsewhere $[13,14]$ the first peak is assigned to hexane and DBT physisorbed in the porous structure of the carbons whereas the second one corresponds to DBT strongly adsorbed due to specific interactions. The assignment of the DTG peaks in PLN and PLS should be done carefully, to avoid misinterpretation with the peaks linked to the decomposition of the surface groups. In samples PL S and PL N, the peaks linked to the decomposition of the carboxylic and sulfonic groups (Figure 2) present for the as-received carbons, disappeared after DBT exposure, and the two desorption peaks due to physisorption and specific interactions are revealed. A similar result was reported in oxidized carbons [13], confirming the important role of acidic groups in the process of DBT reactive adsorption.

Physisorption of DBT is based on dispersive interactions of its polyaromatic structure with the basal planes of the carbon, while another type of adsorption contribution might involve the sulfur atom of DBT molecule and the active sites of the carbon. A plausible mechanism is sulfur-oxygen interactions or, in the case of PLS, disulfur quazibridges formed between the sulfur atom of DBT and the sulfur species of the surface. 
Furthermore, after the DBT exposure the carbons were heated under $\mathrm{N}_{2}$ atmosphere at $600{ }^{\circ} \mathrm{C}$ for 3 or 6 hours and desorption profiles were recorded afterwards. The desorption peaks attributed to DBT present on the surface disappeared, indicating a partial regeneration of the adsorbent upon heating. However, still a large amount of sulfur remained on the carbon after heating at $600^{\circ} \mathrm{C}$, (Table 4), which confirms that there is a large contribution of quasi-irreversible adsorption of DBT. Increasing the heating time from 3 to 6 hours slightly decreased the amount of remaining sulfur although it did not alter the TPD profiles.

Except for the PL sample, the modified carbons have quite high sulfur content after the thermal regeneration, which demonstrates that the irreversible retention of DBT might be linked to the acidic functionalities of both oxidized carbons PLN and PLS. In fact, for PLS, the most acidic one, the amount of sulfur left on the surface is the highest. Since the carbons have the same textural properties, such different behavior can only be attributed to their chemical properties, and particularly to the acidic sites.

Based on these findings, it is evident that the DBT molecule undergoes some kind of secondary reactions (likely polymerization and/or condensation via electrophilic addition reactions) on the surface of acidic carbons and therefore the formed sulfur-based intermediates block the porosity of the carbons. This hypothesis would also explain the high sulfur content of PLS and PLN after heating, as well as their improved adsorption capacity when compared to PL sample. Further studies are ongoing to investigate the nature of the sulfur incorporated to the carbon matrix.

\section{CONCLUSIONS}

Porous carbons obtained from PET waste have promising perspective as cost-efficient adsorbents for deep desulfurization of liquid hydrocarbons. By performing oxidation in mild conditions, we have obtained a series of carbons with different chemical features but similar porous texture.

Introduction of oxygen-containing groups enhanced the performance of the adsorbents in desulfurization of liquid fuels. Adsorption of DBT on activated carbons is governed by two types of contributions: physical and chemical interactions. The correlation between the adsorption capacities and the density of acidic groups on the carbon demonstrated the importance of surface heterogeneity of the carbons for DBT adsorption. 
The large quantities of sulfur retained in the samples even after heating at high temperatures suggest that there is a contribution of quasi-irreversible specific adsorption. The performance of the oxidized carbons, along with the contribution of irreversible retention of DBT on the surface is associated with reactive adsorption involving the acidic sites on the carbon surface, and not to the high treatment temperature. This may occur via breaking of the sulfur-carbon bond in the thiophenic compound, via polymerization and/or condensation reactions in the acidic carbons. Consequently, sulfur-based intermediates are formed and the porosity of the carbons is blocked.

\section{ACKNOWLEDGEMENTS}

The authors thank PSC CUNY for financial support (PSC CUNY 66382-0035). COA thanks MEC for a postdoctoral fellowship (EX2004-0612).

\section{REFERENCES}

[1] D. Jayne, Y.Zhang, S. Haji, C. Erkey. Int. J. Hydrogen Energy 30 (2005) 1287.

[2] EPA-Gasoline RIA, US Environmental Protection Agency, Air and Radiation, EPA420-R-99023, December 1999.

[3] US EPA. EPA 40 CFR Parts 80 and 86, April 2001.

[4] Directive 1998/70/EC of 13 October 1998. Journal L 350, 28/12/ 98. p. 005.

[5] Directive 2003/17/EC of 3 March 2003. Offcial Journal L 076, 22/03/2003. p. 0010.

[6] C.N. Satterfield, Heterogeneous catalysis in industrial practice. New York: McGraw-Hill, 1991, p. 378.

[7] D.D Whitehurst, I. Isoda, I. Mochida, Adv Catal. 42 (1998) 345.

[8] X. Ma, L. Sun, C. Song, Catal Today, 77 (2002) 107.

[9] C. Song, X. Ma, Appl. Catal. B, 41 (2002) 207.

[10] R.T. Yang, A.J. Hernandez-Maldonado, F.H. Yang, Science, 301 (2003) 79.

[11] S. Velu, X. Ma, C. Song, Ind. Eng. Chem. Res. 42 (2003) 5293.

[12] S. Haji, C. Erkey, Ind Eng Chem. Res. 42 (2003) 6933.

[13] C.O. Ania, T.J. Bandosz, Langmuir, 21 (2005) 7752.

[14] C.O. Ania, T.J. Bandosz, Carbon, 2006 (in press) 
[15] J.B. Parra, C.O. Ania, A. Arenillas, F. Rubiera, J.M. Palacios, J.J. Pis, J. Alloys Comp. 379 (2004) 280.

[16] C.O. Ania, J.B. Parra, J.J. Pis, Ads. Sci. Technol. 22 (2004) 337.

[17] A. Derylo-Marczewska, M. Jaroniec, Chem. Scripta, 24 (1984) 239.

[18] J. Olivier, Carbon, 36 (1998) 1469.

[19] C. Giles, T. McEwan, S. Nakhwa, D. Smith, J. Chem. Soc. 1960, 3973.

[20] Z. Jiang, Y. Liu, X. Sun, F. Tian, F. Xun, C. Liang, W. You, C. Han, C. Li, Langmuir 19 (2003) 731.

[21] A. Derylo-Marczewska, A.W. Marczewski, Appl. Surf. Sci. 196 (2002) 264.

[22] S. Haydar, C. Moreno-Castilla, M.A. Ferro-Garcia, F. Carrasco-Marin, J. Rivera-Utrilla, A. Perrard, J.P. Joly, Carbon 38 (2000) 1297.

[23] A.P. Terzyk, J. Colloid Interf. Sci. 268 (2003) 30. 


\section{Figure Captions}

Figure 1. Equilibrium adsorption isotherms of DBT in the series of PET-derived carbons.

Figure 2. TPD/FTIR spectra of $\mathrm{CO}, \mathrm{CO}_{2}$ and $\mathrm{SO}_{2}$ in the series of modified carbons.

Figure 3. Correlation between the adsorption capacity of DBT and the chemical properties of the carbons.

Figure 4. Desorption profiles of the carbons after DBT exposure (sat) and followed by heat treatment at $600{ }^{\circ} \mathrm{C}$ (sat HT).

\section{Table Captions}

Table 1. Fitting parameters of DBT adsorption isotherms to the Langmuir-Freundlich equation and amount of sulfur on the carbons surface after DBT exposure

Table 2. Chemical and textural characteristics of the carbons evaluated from $\mathrm{N}_{2}$ adsorption isotherms at $-196{ }^{\circ} \mathrm{C}$ and the DFT method

Table 3. Comparison of the amount of sulfur adsorbed on the surface of carbons exposed to DBT (from XRF measurements) and after thermal regeneration at $600{ }^{\circ} \mathrm{C}$ 
Figure 1.

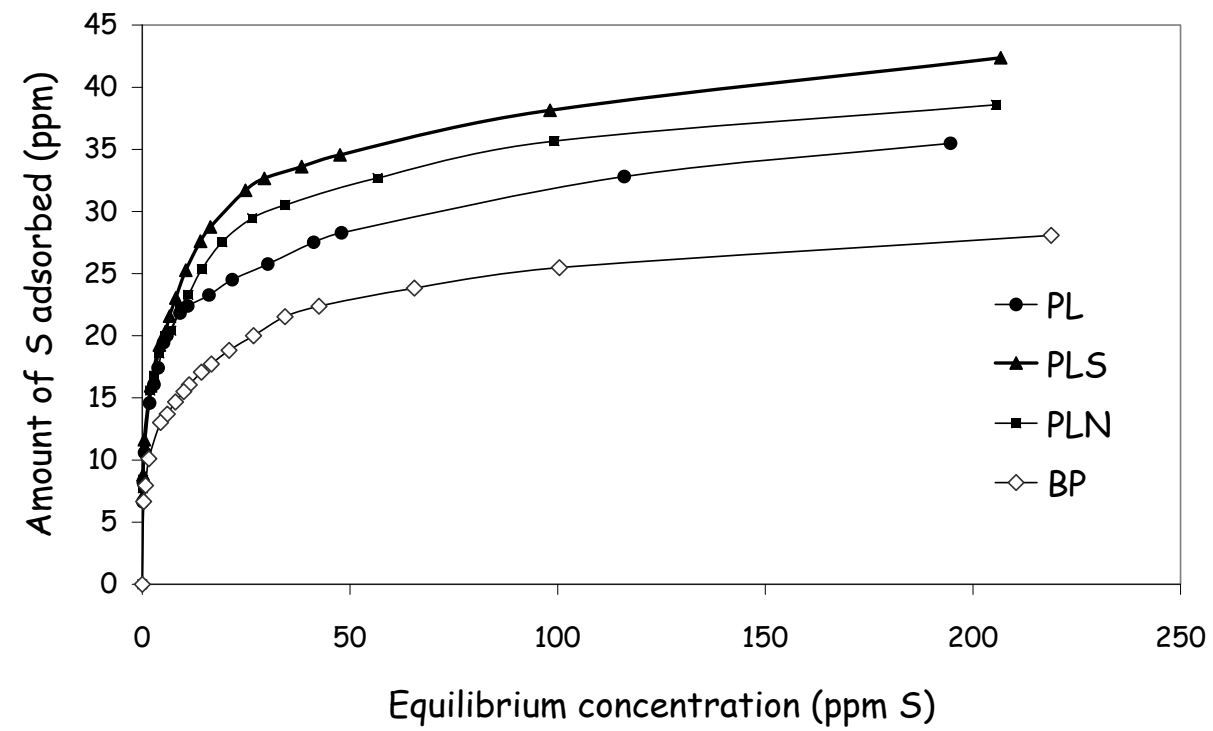


Figure 2.

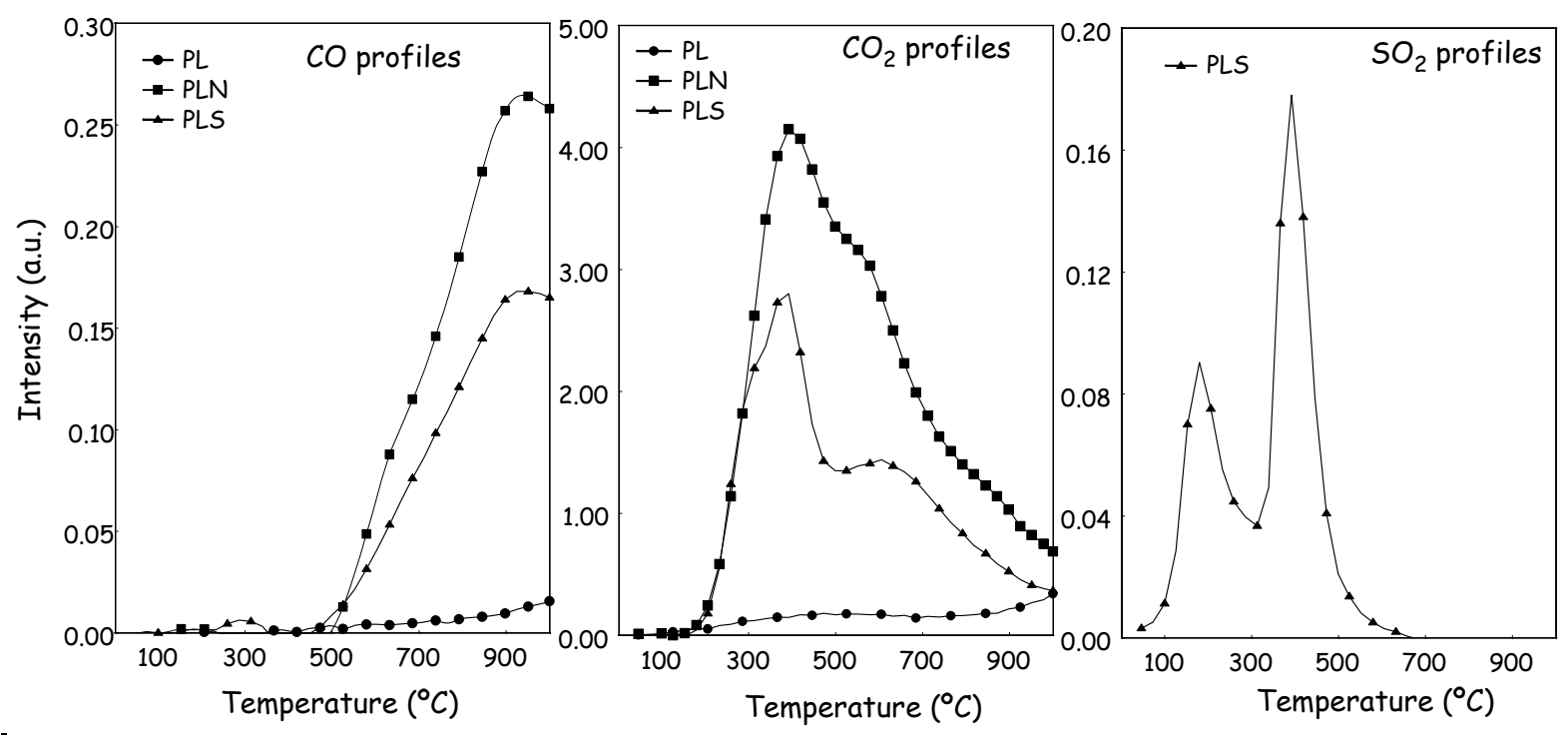


Figure 3.

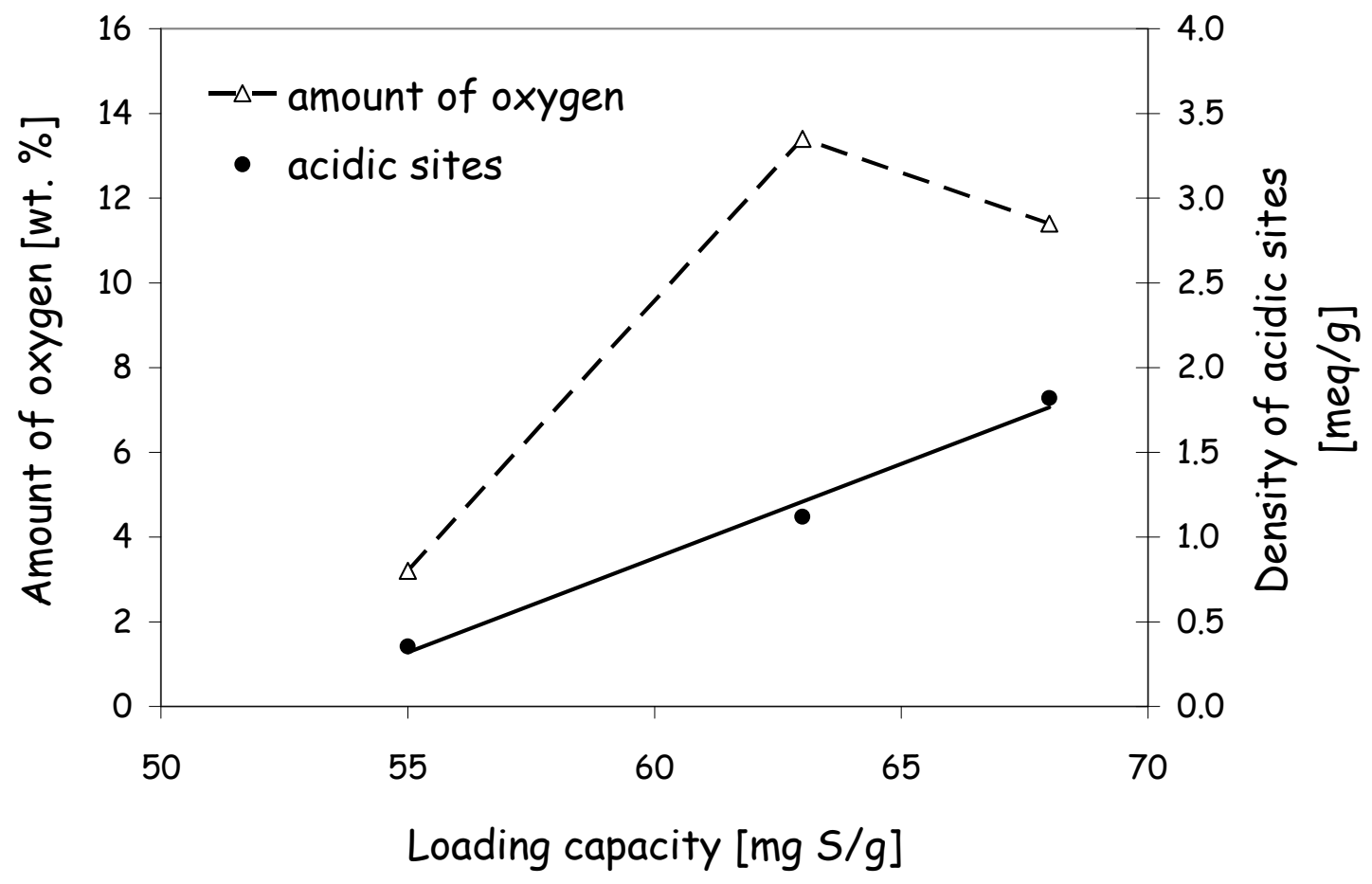


Figure 4.

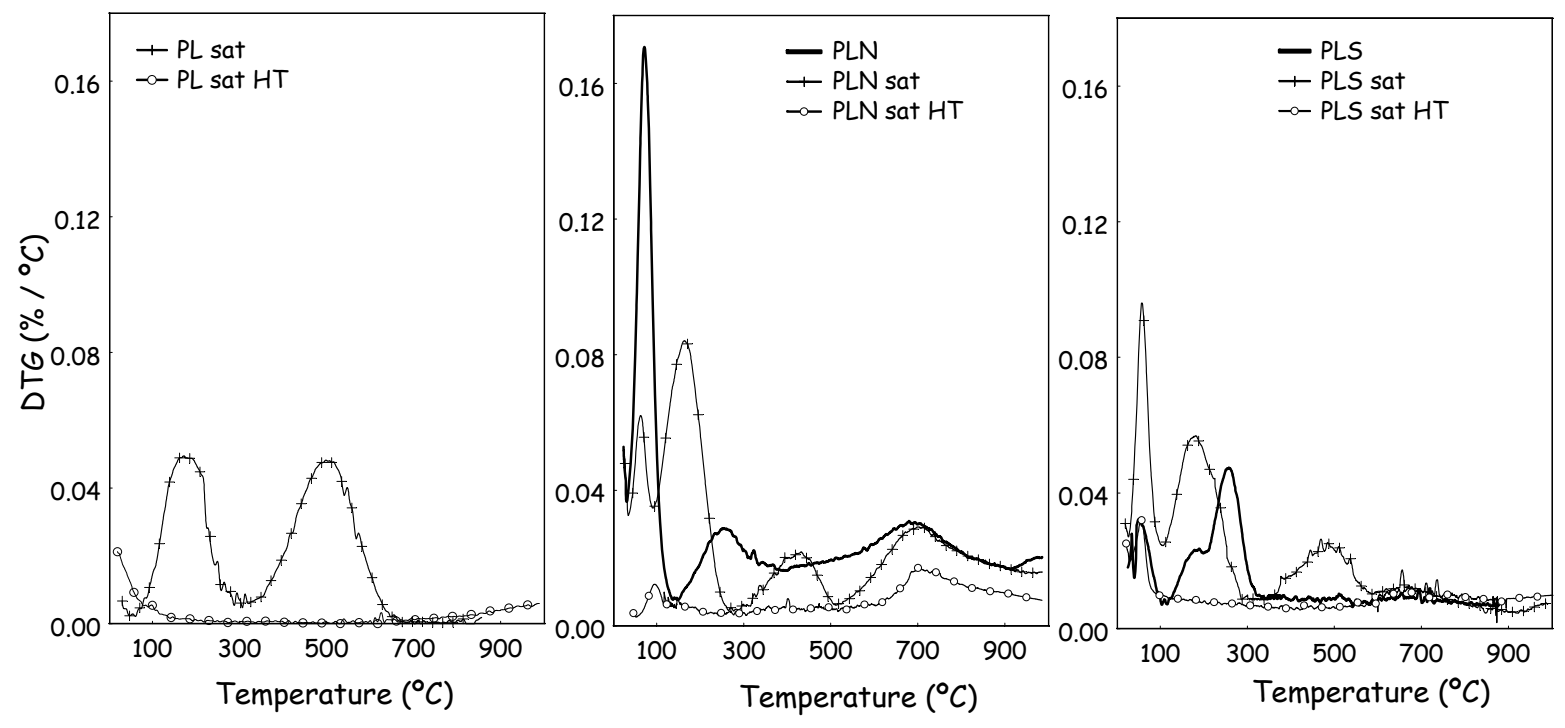


Table 1.

\begin{tabular}{lcccccc}
\hline & $\begin{array}{c}\mathbf{q}_{\mathbf{m}} \\
{[\mathbf{m g ~ S / g}]}\end{array}$ & $\begin{array}{c}\mathbf{q}_{\mathbf{m}} \\
\text { [mg DBT/g] }\end{array}$ & $\mathbf{n}$ & $\mathbf{R}^{2}$ & $\begin{array}{c}\text { \% S } \\
\text { (XRF) }\end{array}$ & $\begin{array}{c}\text { \% S } \\
\text { (expected) } \\
\text { (recalculated from } \\
\text { isotherms) }\end{array}$ \\
\hline PL & 55 & 317 & 0.38 & 0.998 & 5.1 & 5.5 \\
PLS & 68 & 393 & 0.30 & 0.994 & 11 & 6.8 \\
PLN & 63 & 363 & 0.31 & 0.996 & 6.1 & 6.3 \\
BP & 47 & 273 & 0.36 & 0.998 & 4.6 & 4.7 \\
\hline
\end{tabular}

Table 2.

\begin{tabular}{|c|c|c|c|c|c|c|c|c|}
\hline Sample & $\begin{array}{c}S_{\mathrm{BET}} \\
{\left[\mathrm{m}^{2} \mathbf{g}^{-1}\right]}\end{array}$ & $\begin{array}{c}\mathbf{V}_{\text {TOTAL }} \\
{\left[\mathbf{c m}^{3} \mathbf{g}^{-1}\right]} \\
\mathbf{p} / \mathbf{p o}<\mathbf{0 . 9 9}\end{array}$ & $\begin{array}{l}V_{\text {micropore }} \\
{\left[\mathrm{cm}^{3} \mathrm{~g}^{-1}\right]}\end{array}$ & $\begin{array}{l}V_{\text {mesopores }} \\
{\left[\mathbf{c m}^{3} \mathbf{g}^{-1}\right]}\end{array}$ & $\mathbf{p H}$ & $\begin{array}{c}\text { O } \\
\text { [wt. \%] }\end{array}$ & $\begin{array}{c}\text { S } \\
\text { [wt. \%] }\end{array}$ & $\begin{array}{c}\text { Density of } \\
\text { acidic sites } \\
\text { [meq/g] }\end{array}$ \\
\hline PL & 1152 & 0.516 & 0.361 & 0.060 & 6.9 & 3.2 & n.d. & 0.354 \\
\hline PLS & 1063 & 0.489 & 0.331 & 0.069 & 2.1 & 11.4 & 4.5 & 1.820 \\
\hline PLN & 1063 & 0.470 & 0.330 & 0.049 & 3.5 & 13.4 & n.d. & 1.120 \\
\hline
\end{tabular}

Table 3.

\begin{tabular}{lccc}
\hline \% Sulfur (XRF) & PL & PLS & PLN \\
\hline as-received & n.d. & 4.5 & n.d. \\
after DBT exposure & 5.1 & 11 & 6.1 \\
after heating $\mathbf{6 0 0}^{\circ} \mathbf{C} \mathbf{3} \mathbf{~ h}$ & 1.1 & 7.9 & 2.5 \\
after heating $\mathbf{6 0 0}^{\circ} \mathbf{C ~} \mathbf{6 ~ h}$ & 0.6 & 7.4 & 2.2 \\
\hline
\end{tabular}

